\section{A familial case of Takayasu arteritis: HLA-related genetic susceptibility and efficacy of anti-TNFa treatment in two italian siblings}

\begin{abstract}
The aetiology of Takayasu Arteritis (TA) remains poorly understood. An autoimmune origin is the main hypothesis, suggesting an abnormal immune response against unknown antigens present on vessel walls. The genetic contribution is also considered relevant in the TA pathogenesis and is mainly supported by the association with specific patterns of HLA. In Japan and other Asian countries the allele B5, and its split B52, is the most frequent and associates with a severe disease and worse prognosis. Other possible alleles of susceptibility are B39, DRB1*0405 and non-HLA loci, related to cytokine expression (IL6 and IL12 and TNFa). Most of reported familial studies concern Asian patients.
\end{abstract}

Here we describe the case of a young Italian woman and his brother, both affected by TA, who shared similar clinical features and the same HLA pattern, especially the haplotype HLA-B52 and other alleles (A2, B44, DRB1-14 and DRB1-16). They were both treated with infliximab, after the failure of standard therapy with methotrexate and corticosteroids, achieving a remission of the disease, confirmed by imaging. This familial case may contribute to remark the importance of genetic pattern in the onset of the disease and the efficacy of anti-TNFa treatment in TA patients.

Keywords: takayasu's arteritis $\cdot$ genetic susceptibility $\bullet$ familial case

\section{Background}

Takayasu Arteritis (TA) is a rare vasculitis affecting large vessels, especially aorta and its main branches. Vessel inflammation leads to arterial thickening, fibrosis, stenosis and progressive occlusion or to dilatation, aneurysm and thrombus formation [1]. Clinical manifestations are heterogeneous, ranging from an asymptomatic disease to catastrophic neurological manifestations. Usually the disease begins with non-specific inflammatory symptoms including fever, fatigue, arthromyalgias and weight-loss, followed by a chronic phase characterized by vascular complications and more specific features: reduced pulses, vessel murmurs, upper limbs claudication [2].

TA has a large female predominance (9:1 female to male ratio), typically develops in the second or third decade and is more frequently reported in Japan, East Asia, India, and Mexico [2,3].

The aetiology of TA remains poorly understood. An autoimmune origin is the main hypothesis, suggesting an abnormal immune response against unknown antigens present on vessel walls. This mechanism might involve inflammatory cells, especially monocytes and T-cells, and proinflammatory cytokines such as IL6 and TNFa.
The genetic contribution to the disease pathogenesis was also considered by several authors since the sixties and following years. It is mainly supported by the association with a specific pattern of HLA class I and II and with non-HLA genes involved in immune response (Table 1) [4-9].

The main reported association is with HLA-B5 and especially with its split B52 [8-11]. The allele B51 is also considered a possible gene of susceptibility but the evidence is weaker [12].

Yoshida et al. [4] reported an increased frequency of $B$ 39.2, which shares the peptide binding epitope with HLA-B52. Other alleles, such as DRB1, DR2, DQ1, are also suggested to be associated with TA [8].

North American studies found an association between TA and DR4 and MB3 [13], while in Italy Colombo et al. [14] reported an increased prevalence of HLA-DRB1*0405 which was associated with early-onset of vasculitis.

Recent studies, involving Turkish and North American patients, identified a correlation between TA and non HLA-correlated genes, such as IL6, RPS9/LILRB3 (encoding inhibitory
Myriam Facchinetti*,

Pierantonio Ostuni \& Gabriella Cardinale

U.O.S. Rheumatology, S. Antonio Hospital ULSS6 Padua, Italy

${ }^{*}$ Author for correspondence:

f.myriam19@gmail.com 
Table 1. Main alleles/loci associated with TA observed in studies of different ethnical groups.

\begin{tabular}{|c|c|c|c|}
\hline & Alleles/loci & Observed population & Type of association with TA \\
\hline \multirow{7}{*}{ HLA class I } & $A(A 10)$ & Japan & possible protective role \\
\hline & B39 & Japan, Mexico & susceptibility, linked to B52 \\
\hline & B51 & Japan, Turkey & susceptibility (weak) \\
\hline & $\mathrm{B} 52^{*} 01$ & $\begin{array}{c}\text { Japan, China, North America, } \\
\text { Turkey, India, Mexico }\end{array}$ & $\begin{array}{l}\text { susceptibility (strong), severe disease, } \\
\text { poor prognosis }\end{array}$ \\
\hline & B67 & Japan & susceptibility (weak) \\
\hline & $\mathrm{Cw}^{*} 07$ & Europe, America, Turkey & possible protective role \\
\hline & $\mathrm{CW}^{*} 12$ & Europe, America, Asia & susceptibility \\
\hline \multirow{2}{*}{ HLA class II } & DRB $1 * 0405$ & North America, Europe & susceptibility, early-onset disease \\
\hline & $\mathrm{DRB} 1 * 07$ & China, Japan & susceptibility \\
\hline \multirow{4}{*}{ non-HLA } & IL6 & America, Turkey & susceptibility \\
\hline & RSP9/LILRB3 & America, Turkey & susceptibility \\
\hline & IL12B & Japan & $\begin{array}{c}\text { susceptibility, possible resistance to } \\
\text { therapy }\end{array}$ \\
\hline & $\begin{array}{l}\text { TNFa 308A/G } \\
\text { polymorphism }\end{array}$ & Japan, China & susceptibility \\
\hline
\end{tabular}

immunoglobulin-like receptor proteins), and an intergenic locus on chromosome $21 \mathrm{q} 22$ and IL12B region $[15,16]$. The association with TNF-a-308A/G polymorphism is also reported in Japanese works [12].

A possible association with genetic causes is supported by familial cases of TA, most of them are described in Asian countries [17-23].

Standard treatment of TA is corticosteroid therapy. Traditional immunosuppressant agents as methotrexate are often used but their effectiveness is variable and incomplete. Several patients with refractory disease are now treated with biologic drugs. Anti-TNFa agents and more recently anti-IL6 showed the best efficacy in severe cases of TA [24-27].

\section{A familial case report}

We report the case of a young Italian woman and his brother, both affected by TA. Their mother is healthy and their father died because of a brain neoplasm.

Case 1

An Italian young woman referred to our Clinic in October 2011, when she was 27 years old, with persistent carotidynia, weakness and dyspnea on exertion. She complained left lateral cervical and left shoulder pain in the previous 6 years. At clinical examination she had hyposphigmia with different blood pressure values between her right and left arm, a mild left carotid murmur and a marked diastolic murmur in mesocardial area.
The diagnosis of TA was confirmed by imaging: the arterial US showed, on the left side, marked and widespread thickening of the whole course of common carotid, as well as of internal and external carotid, determing a severe stenosis of the vessel especially at the carotid bifurcation. A PETCT revealed mild but diffuse hypermetabolism on the left side of ascending aorta and the left common carotid. Echocardiography showed mitral prolapse and moderate aortic valve failure.

She had elevated C-Reactive Protein (CRP) and Erythrocyte Sedimentation Rate (ESR) values. Autoantibodies, including antiphospholipid antibodies, were absent. High doses corticosteroid treatment was started, associated with methotrexate $15 \mathrm{mg}$ weekly and low dose aspirin, with a rapid improvement of symptoms and inflammatory markers. About 6 months later the arterial US showed worsening of the vascular disease, with occlusive stenosis of left carotid and subclavian arteries, although ESR and CRP remained normal, and she required a dose of corticosteroid not inferior to $25 \mathrm{mg}$ daily.

A screening in order to start anti-TNFa therapy was performed and in August 2012 infliximab originator (Remicade) $300 \mathrm{mg}$ was initiated. After the induction dose, she received an infusion every 4 weeks during the first 6 months and then the dosing interval was gradually prolonged until 8 weeks. The biologic treatment, associated with methotrexate $10 \mathrm{mg}$ and low dose corticosteroid (5-7.5 mg daily), was effective in controlling the disease progression and symptoms. 


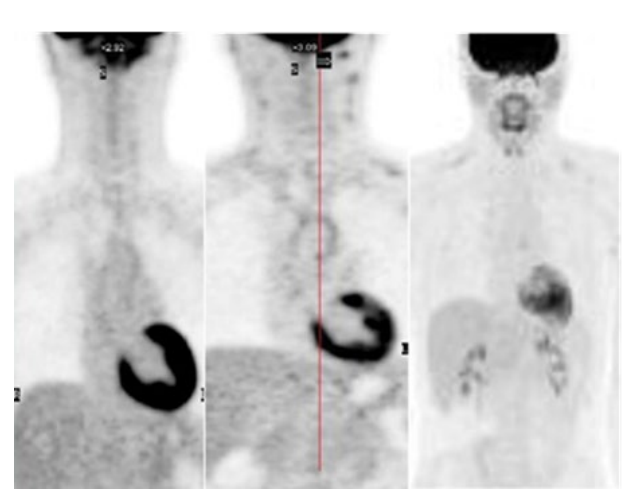

Figure 1. progression of PET-CT images in the second patient. 1A: involvement of the whole thoracic aorta, the arch and the discending tract and of the sovraortic branches, especially the right common carotid (PET- CT performed on 2015). 1B: worsening with involvement of the aortic bulb (PET-CT on 2016). 1C: significant improvement in all affected vessels (PET-CT on 2017).

Now, seven years later, she is clinically stationary, presenting rare transient episodes of left arm paresthesias and carotidodinì which require short courses with minimal increase of corticosteroid doses.

A recent arterial US showed very mild decrease of the stenosis of left carotid branches, but in the last PET-CT examination, performed in January 2017, previous hypermetabolism of the aorta walls has completely disappeared.

\section{Case 2}

Her brother started to complain neck pain when he was 18 years old. He referred to our clinic some years later and he was evaluated in the suspect of the same disease affecting his sister. $\mathrm{He}$ also had a blood pressure gap superior of 20 $\mathrm{mmHg}$ between his two arms and inflammation markers were elevated. The arterial US showed thickening of the whole right common carotid, determing a stenosis of $80 \%$, of the whole left common carotid and left internal carotid, with stenosis of $60 \%$ and $35 \%$ respectively. The PETCT showed significant hypermetabolism of the whole thoracic aorta, especially of the ascending part, the arch and the discending tract until D10, and of the sovraortic branches, especially the right common carotid (Figure 1A).

On the basis of these findings, the diagnosis of TA was confirmed in june 2014. The young patient was treated with high dose corticosteroid infusions (750 mg for 3 days every month) during about 10 months, adding methotrexate until the dose of $20 \mathrm{mg}$, with transient benefit. He continued during the next six months with methotrexate $15 \mathrm{mg}$ weekly and mild doses of oral corticosteroid (20-25 mg daily). CRP and ESR values remained high and carotidodinìa appeared again. In January 2016 he performed a PET-CT which showed a progression of the thickening involving the aortic bulb (Figure 1B) and the arterial US was unmodified, so he started infusions of infliximab biosimilar (Inflectra) 300 mg monthly.

After 4 months of therapy, the patient presented clinical and serological improvement, disappearing of neck pain and normalization of inflammation markers. At the arterial US, carotid involvement was unmodified and the vessels of both arms and legs were normal. After 2 years clinical improvement persists and the biologic therapy is well tolerated. The last PETCT (January 2017) also reported a significant decrease of hypermetabolism of the thoracic aorta and upper vessels (Figure 1C).

HLA typing: We acquired a written agreement of both affected patients for genetic examination. Serum samples of the two siblings were sent to the Immunotransfusional Center and HLA typing was determined. According to literature findings about the main genetic patterns associated with TA, we asked our laboratory to type the following loci: HLA-A, HLA-B and HLA-DR. The two siblings shared the following haplotypes: HLA-A2, B44, B52, DRB1-14 and DRB1-16.

\section{Discussion}

The autoimmune hypothesis of TA onset is supported by histo-pathological findings, as inflammatory cells in arterial walls, and indirectly by efficacy of biologic anti-cytokine drugs, especially anti-TNFa and anti-IL6 agents.

The importance of genetics is suggested by ethnical distribution of affected patients and by several findings of association with HLA alleles. A large contribution is supported by familial cases, which are reported predominantly in Asia. In a review by Morishita, Rosendahl and Brogan, 31 different familial cases were found, most of them in Japan [21]. A particular case concerns a Pakistani family, where five out of seven siblings were affected with TA [23].

Our two young patients shared very similar clinical features, with light difference of vessel involvement, and the same HLA pattern, especially the haplotype B52, which represents one of the main alleles associated with TA, according to several studies. 
The allele HLA-B52 is the most frequently and strongly associated with TA in Japan and other Asian countries [8,9] and relates with worse clinical manifestations, higher rate of vascular complications and poor prognosis [10,11]. According to literature findings, our patients presented severe features suggesting a bad prognosis: the disease onset was underhand but the progression of thickening of the arterial walls was rapid and diffuse, leading to severe carotid occlusion and involving also the aortic bulb in case 2. Furthermore, the response to standard therapy was transient and incomplete, so both patients needed the introduction of a biologic agent.

Other alleles are observed in association to TA, but with less agreement among authors and more ethnical differences, for example allele DRB ${ }^{*} 0405$ is associated to the disease in Europe and North America and DRB1*07 in Asia. Some haplotypes HLA A and C might have a protective role (Table 1 ). Efficacy of anti$\mathrm{TNF} \alpha$ treatment in our patients was very similar, rapid and persistent, allowing in both cases the reduction of steroid doses, although the young woman started infliximab infusions several years after the disease onset. Clinical improvement was associated with a relevant change in PETTC findings in both patients, suggesting the inhibition of inflammatory disease and vessel damage progression. No adverse events related with infusions were reported.

We observed no difference of response and tolerability between the young woman, treated with infliximab originator (Remicade), and her brother, treated with infliximab biosimilar (Inflectra). Effectiveness of infliximab in TA patients is reported in the literature [24-26]. It could be interesting to study also the relationship between genetic haplotype and response to therapy, in order to identify subset of patients with a pattern refractory to standard treatments who may benefit from anti-TNF $\alpha$ agents.

We chose the biologic therapy on the basis of literature findings and, especially in the second case, of our previous experience. Nowadays, more evidence of efficacy of anti-IL6 drugs in TA is available [27], providing a valid therapeutic alternative.

\section{Conclusion}

According to the literature, we observed in our familial case the association between TA and a specific genetic pattern (HLA-B52). This finding may contribute to remark the importance of genetics in the onset of the arteritis and of a severe pattern. We also may remark the effectiveness of anti-TNFa in our patients. A larger number of studies is required, however, to define better the role of genetic in patients affected with TA.

\section{References}

1. Kerr GS, Hallahan CW, Giordano J, et al. Takayasu's arteritis. Ann. Intern. Med. 120(11), 919-929 (1994).

2. Hall S, Barr W, Lie JT, et al. Takayasu arteritis. A study of 32 North American patients. Medicine (Baltimore). 64(2), 89-99 (1985).

3. Shimizu K, Sano K. Pulseless disease. J. Neuropathol. Clin. Neurol. 1(1), 37-47 (1951).

4. Yoshida M, Kimura A, Katsuragi K, et al. DNA typing of HLA-B gene in Takayasu's arteritis. Tissue. Antigens. 42(2): 87-90 (1993).

5. Kimura A, Kitamura H, Date $\mathrm{Y}$, et al. Comprehensive analysis of HLA genes in Takayasu arteritis in Japan. Int. J. Cardiol. 54(Suppl 1), S61-S69 (1996).

6. Kimura A, Ota M, Katsuyama Y, et al. Mapping of the HLA-linked genes controlling the susceptibility to Takayasu's arteritis. Int. J. Cardiol. 75 (Suppl1), S105S110 (2000).

7. Sharuhan-Direskeneli G, Hughes T, Aksu $\mathrm{K}$, et al. Identification of multiple genetic susceptibility loci in Takayasu arteritis. Am. J. Hum. Genet. 93(2), 298-305 (2013)

8. Terao C. Revisited HLA and non-HLA genetics of Takayasu arteritis-where are we? J. Hum. Genet. 61(1), 27-32 (2016).

9. Renauer P, Sawalha AH. The genetics of Takayasu arteritis. Presse. Med. 46 (7-8), e169-e187 (2017)

10. Kasuya K, Hashimoto Y, Numano F. Left ventricular dysfunction and HLA-Bw52 antigen in Takayasu arteritis. Heart. Vessels. 7(suppl 1), 116-119 (1992).

11. Origuchi T, Fukui S, Umeda M, et al. The severity of Takayasu Arteritis is associated with the HLA-B52 allele in Japanese patients. Tohoku. J. Exp. Med. 239 (1), 6772 (2016). 
12. Chen S, Luan H, Li L, et al. Relationship of HLA-B*51 and HLA-B*52 alleles and $\mathrm{TNF} \alpha-308^{\circ} / \mathrm{G}$ polymorphism with susceptibility to Takayasu arteritis: a metaanalysis. Clin. Rheumatol. 36(1), 173-181 (2017).

13. Volkman DJ, Mann DL, Fauci AS. Association between Takayasu's arteritis and a B-cell alloantigen in North Americans. $N$. Engl. J. Med. 306, 464-465 (1982).

14. Colombo G, Compasso S, Vanoli M. HLA and susceptibility to Takayasu's arteritis in the Italian population. Eur. J. Immunogen. 25(suppl 1), S64 (1998).

15. Renauer PA, Saruhan-Direskeneli G, Coit P. Genome-wide association study identifies susceptibility loci in IL6, RPS9/LILRB3, and an intergenic locus on chromosome 21q22 in Takayasu Arteritis. Arthritis. Rheumatol. 67, 1361-1368 (2015).

16. Nakajima T, Yoshifuji H, Shimizu M, et al. A novel susceptibility locus in the IL12B region is associated with the pathophysiology of Takayasu arteritis through IL-12p40 and IL-12p70 production. Arthritis. Res. Ther. 19(1), 197 (2016).

17. Hirsch MS, Aikat BK, Basu AK. Takayasu's arteritis: report of five cases with immunologic studies. Bull. Johns. Hopkins. Hosp. 115, 29-64 (1964).

18. Numano F, Isohisa I, Kishi U, et al. Takayasu's disease in twin sisters: possible genetic factors. Circulation. 58, 173-77 (1978).

19. Enomoto S, Iwasaki $Y$, Bannai $S$, et al. Takayasu's disease in twin sisters. Jpn. Heart. J. 25, 147-52 (1984).
20. Kodama K, Kida O, Morotomi Y, et al. Male siblings with Takayasu's arteritis suggest genetic etiology. Heart. Vessels. 2, 51-54 (1986).

21. Morishita KA, Rosendhal K, Brogan PA. Familial Takayasu arteritis a pedriatric case and a review of the literature. Pediatr. Rheumatol. Online. J. 9, 6 (2011).

22. Deniz A, Yildiz F, Aktas H, et al. Familial Takayasu arteritis in a mother and daughter, A report of two cases. Herz. 38, 93-96 (2013).

23. Jeeva I, Sajid J. Atypical Takayasu arteritis: A family with five affected siblings. Med. Sci. Monit. 13(8), CS101-105 (2007).

24. Hoffman GS, Merkel PA, Brasington RD, et al. Anti-tumor necrosis factor therapy in patients with difficult to treat Takayasu arteritis. Arthritis. Rheum. 50(7), 2296-304 (2004).

25. Comarmond C, Plaisier E, Dahan K, et al. Anti-TNFa in refractory Takayasu's arteritis: cases series and review of the literature. Autoimmun. Rev. 11(9), 678-684 (2012).

26. Mekinian A, Comarmond C, RescheRigon $\mathrm{M}$, et al. Efficacy of biologicaltargeted treatments in Takayasu Arteritis: multicenter, retrospective stydy of 49 patients. Circulation. 132(18), 1693-1700 (2015).

27. Mekinian A, Resche-Rigon M Comarmond C, et al. Efficacy of tocilizumab in Takayasu arteritis: Multicenter retrospective study of 46 patients. J Autoimmun. 91, 55-60 (2018). 